\title{
THE SATURATION NUMBER FOR THE LENGTH OF DEGREE MONOTONE PATHS
}

\author{
YAIr CARO ${ }^{1}$, Josef LAURI ${ }^{2}$ \\ AND \\ Christina ZARB ${ }^{2}$ \\ ${ }^{1}$ Department of Mathematics \\ University of Haifa-Oranim, Israel \\ ${ }^{2}$ Department of Mathematics \\ University of Malta, Malta \\ e-mail: yacaro@kvgeva.org.il \\ josef.lauri@um.edu.mt \\ christina.zarb@um.edu.mt
}

\begin{abstract}
A degree monotone path in a graph $G$ is a path $P$ such that the sequence of degrees of the vertices in the order in which they appear on $P$ is monotonic. The length (number of vertices) of the longest degree monotone path in $G$ is denoted by $m p(G)$. This parameter, inspired by the well-known ErdösSzekeres theorem, has been studied by the authors in two earlier papers. Here we consider a saturation problem for the parameter $m p(G)$. We call $G$ saturated if, for every edge $e$ added to $G, m p(G+e)>m p(G)$, and we define $h(n, k)$ to be the least possible number of edges in a saturated graph $G$ on $n$ vertices with $m p(G)<k$, while $m p(G+e) \geq k$ for every new edge $e$.

We obtain linear lower and upper bounds for $h(n, k)$, we determine exactly the values of $h(n, k)$ for $k=3$ and 4 , and we present constructions of saturated graphs.
\end{abstract}

Keywords: paths, degrees, saturation.

2010 Mathematics Subject Classification: 05C07, 05C35, 05 C38.

\section{REFERENCES}

[1] B. Bollobás, Extremal Graph Theory (Dover Publications, New York, 2004).

[2] Y. Caro, J. Lauri and C. Zarb, Degree monotone paths, ArXiv e-prints (2014) submitted. 
[3] Y. Caro, J. Lauri and C. Zarb, Degree monotone paths and graph operations, ArXiv e-prints (2014) submitted.

[4] J. Deering, Uphill and downhill domination in graphs and related graph parameters, Ph.D. Thesis, ETSU (2013).

[5] J. Deering, T.W. Haynes, S.T. Hedetniemi and W. Jamieson, Downhill and uphill domination in graphs, (2013) submitted.

[6] J. Deering, T.W. Haynes, S.T. Hedetniemi and W. Jamieson, A Polynomial time algorithm for downhill and uphill domination, (2013) submitted.

[7] M. Eliáš and J. Matoušek, Higher-order Erdös-Szekeres theorems, Adv. Math. 244 (2013) $1-15$.

doi:10.1016/j.aim.2013.04.020

[8] P. Erdős, A. Hajnal and J.W. Moon, A problem in graph theory, Amer. Math. Monthly 71 (1964) 1107-1110. doi:10.2307/2311408

[9] P. Erdős and G. Szekeres, A combinatorial problem in geometry, Compos. Math. 2 (1935) 463-470.

[10] J.R. Faudree, R.J. Faudree and J.R. Schmitt, A survey of minimum saturated graphs, Electron. J. Combin. 18 (2011) \#DS19.

[11] T.W. Haynes, S.T. Hedetniemi, J.D. Jamieson and W.B. Jamieson, Downhill domination in graphs, Discuss. Math. Graph Theory 34 (2014) 603-612. doi:10.7151/dmgt.1760

[12] L. Kászonyi and Zs. Tuza, Saturated graphs with minimal number of edges, J. Graph Theory 10 (1986) 203-210. doi:10.1002/jgt.3190100209

Received 14 September 2014

Revised 6 November 2014

Accepted 14 November 2014 